\title{
EL CAMBIO EDUCATIVO ANTE LA INNOVACIÓN TECNOLÓgICA, LA PEDAgOGÍA DE LAS COMPETENCIAS Y EL DISCURSO DE LA EDUCACIÓN EMOCIONAL. UNA MIRADA CRÍTICA ${ }^{1}$
}

\author{
The educational change in front of the technological \\ innovation, the pedagogy of competences and the discourse \\ of the emotional education. A critical approach.
}

Jordi SOLÉ BLANCH

Universitat Oberta de Catalunya. España.

jsolebla@uoc.edu

bttp://orcid.org/0101-1213-0917-371X

Fecha de recepción: 18/06/2019

Fecha de aceptación: 25/09/2019

Fecha de publicación en línea: 01/01/2020

RESUMEN

El objetivo de este artículo radica en analizar las presiones que recibe el sistema educativo para adaptarlo a las necesidades del mundo económico. Para ello se examinan las direcciones que está tomando en tres planos diferentes. En primer lugar, se aborda el papel de la innovación educativa vinculada al desarrollo de aplicaciones tecnológicas, que no solo está configurando nuevos modelos de enseñanza y aprendizaje, sino también oportunidades de negocio inspiradas en la industria del entretenimiento, amenazando así la continuidad del sistema educativo tal y como lo conocemos hoy en día. En segundo lugar, se examina el enfoque pedagógico de las competencias que, más allá de promover cambios en la cultura escolar, favorece

1. Este artículo da continuidad al trabajo iniciado por el autor en el marco del Seminario Internacional PEI Abierto «Aprender a imaginarse» organizado por el Programa de Estudios Independientes del MACBA en el año 2017. La ponencia que se presentó en el seminario se publicará en una obra colectiva de la colección de ensayos MACBA "Et AL", coeditada con Arcadia. 
el desarrollo de una visión pragmática y económica de la educación. Finalmente, y partiendo del discurso en torno a la educación competencial y la teoría de las inteligencias múltiples, se analiza el papel que está tomando el fomento de las competencias emocionales en educación a partir de la influencia de la psicología positiva y la industria de la felicidad. Las conexiones entre estos tres planos nos permiten mostrar hasta qué punto contribuyen a configurar subjetividades funcionales a la racionalidad neoliberal que nos gobierna. Ante estas tendencias, cabe reivindicar la renovación del compromiso pedagógico con las nuevas generaciones, que no puede quedar reducido a una formación a la carta dirigida al desarrollo profesional ni a una cultura educativa interesada tan solo en las competencias emocionales y el fomento del espíritu emprendedor. En oposición a las tendencias pedagógicas actuales que defienden un modelo de ciudadanía individualista y psicológico, la perspectiva que sostenemos en este artículo — siguiendo a Hannah Arendt- entiende ese compromiso pedagógico como el esfuerzo permanente por poner en contacto al sujeto con la cultura, el deseo de saber y la apertura a un mundo común.

Palabras clave: innovación educativa; nuevas tecnologías; competencias; educación emocional; contenidos escolares.

\section{ABSTRACT}

The purpose of this article is to analyse the pressures placed upon the education system in order for it to adapt to the needs of the economic world. Therefore, we shall investigate what directions that system is taking nowadays on three different levels. First, we analyze the role of educational innovation linked to the development of technological applications, which is not only configuring new teaching and learning models, but also business opportunities inspired by the entertainment industry, thus threatening the continuity of the educational system and as we know it today. Secondly, we examine the pedagogical approach to competencies that, beyond promoting changes in school culture, favors the development of a pragmatic and economic vision of education. Finally, and based on the discourse about competency education and the theory of multiple intelligences, we analyze the role that the promotion of emotional competencies in education is taking from the influence of positive psychology and the happiness industry. The connections between these three levels underline how it contributes to configure the functional subjectivities according to the neoliberal rationality that governs us. Facing these tendencies, it appears necessary to claim the renewal of the pedagogical commitment with the new generations. This commitment has to provide more than only a free election of a formative menu for the professional development or the development of an educational culture interested mainly in the emotional competencies and the promotion of the spirit of entrepreneurship. In opposition to the current pedagogical tendencies that only encourage a model of individualistic and psychological citizenship, we claim - like Hannah Arendt - a pedagogical commitment relating the subject with culture, the desire of knowledge and the opening to a common world.

Keywords: educational innovation; new technologies; competences; emotional education; school contents. 


\section{INTRODUCCIÓN}

En las últimas décadas, el sistema educativo ha sido objeto de enormes presiones para adaptarlo a las necesidades del mundo económico. Tal y como analizó Christian Laval (2004) en su conocido ensayo La escuela no es una empresa, la política educativa dominante en el mundo actual busca su eficacia y, para ello, debe asimilar la educación a la producción de capital humano a fin de aumentar la competitividad económica en el marco de un mundo globalizado. Sin ir más lejos, las últimas leyes educativas de nuestro país se expresan en estos términos.

Es conocida la jerga que aboga por transformar los sistemas escolares de acuerdo con el nuevo espíritu del capitalismo. Una enorme literatura administrativa defiende la necesidad de ofrecer una formación integral que permita a las nuevas generaciones enfrentar los desafíos de nuestro tiempo potenciando su talento, capacidades y competencias, mientras se hace hincapié en el fomento del espíritu emprendedor, necesario para hacer frente a las demandas de empleo del futuro. Asimismo, y en nombre de la mejora de la calidad de la enseñanza, los nuevos modelos de la gobernanza educativa están fijando su atención en la obtención de resultados y la rendición de cuentas como objetivo principal de todo el sistema educativo.

En este contexto, la innovación educativa, que llega acompañada de una gran efervescencia mediática, aparece como la palanca de las reformas que permitirán superar las limitaciones de la formación que hoy ofrecen la mayoría de escuelas, institutos y universidades. Imbuida de una fuerte impronta empresarial, la innovación «está presente en todo aquello que se quiere destacar, vinculándose siempre al éxito. Innovar es crear, es desarrollar novedad, lo moderno, [...]»(Bermejo, 2018, p. 298), aunque no siempre suponga una mejora, sobre todo en el contexto educativo. En este artículo analizaremos las direcciones que está tomando en tres planos diferentes: la innovación vinculada a las aplicaciones tecnológicas, el enfoque pedagógico de las competencias y la educación emocional.

Sin duda, las tecnologías aplicadas a la educación se han convertido en el instrumento más eficaz para transformar el modelo pedagógico de nuestras instituciones educativas. Más allá de la mejora del ejercicio docente y de facilitar un mejor acceso a la información, las iniciativas innovadoras vinculadas a las tecnologías apuntan a horizontes mucho más ambiciosos. En clave didáctica y curricular, proporcionan, entre otras cosas, un contexto favorable para dar el paso definitivo hacia el llamado currículum por competencias, siguiendo la agenda política europea. Un currículum que ha hallado en el discurso en torno a la educación emocional, avalado por la ciencia y el mundo académico, el aliado necesario para configurar subjetividades funcionales a la racionalidad neoliberal que nos gobierna. Veamos cómo se vincula todo ello y de qué manera podemos recuperar hoy el compromiso pedagógico con las nuevas generaciones. 


\section{LA INNOVACIÓN TECNOLÓGICA}

Buena parte del discurso en torno a la innovación educativa ha quedado reducido a una cuestión metodológica, centrando toda la atención en una didáctica instrumental vacía de reflexión crítica y desconectada de los entornos socioculturales de los centros educativos, así como de cualquier aspiración política. Entre las múltiples propuestas de ese culto a la innovación, brilla con luz propia el papel de la tecnología, que se ha convertido en una auténtica ideología. Tal y como sucede en muchos ámbitos (Morozov, 2015), en ella se espera hallar la solución a los desafíos educativos de nuestra época.

Hace años que las multinacionales tecnológicas están invirtiendo mucho dinero en el diseño del modelo pedagógico del futuro. En la actualidad, ya existen múltiples aplicaciones y herramientas digitales que, como las app de Google, están siendo utilizadas en los diferentes niveles del sistema educativo. Pero esto no ha hecho más que empezar. Solo hay que observar la evolución de la industria del entretenimiento para observar hasta qué punto la innovación pedagógica en marcha ha encontrado su inspiración en la filosofía de Netflix, Uber y Pokemon Go, es decir, formación a la carta, economía colaborativa y gamificación como base de una educación tecnocrática capaz de extenderse al ritmo de las plataformas de internet y sus canales de comunicación y distribución.

Poco a poco se va consumando el desplazamiento de la escuela, desbordada desde hace mucho tiempo por múltiples contextos de aprendizaje. No solo tiene que ver con la consabida certeza de que la enseñanza, tal y como pronosticó McLuhan (1986), tiene lugar fuera de la escuela, donde se multiplican las oportunidades formativas, la información, los conocimientos, los dispositivos, etc., y se diluyen las fronteras entre enseñanza y aprendizaje, sino, sobre todo, con el modelo de negocio que se avecina en el contexto del capitalismo cognitivo, cuya oferta educativa está destinada a proporcionar un sistema de enseñanza programada y multimodal dispuesta a cumplir, esencialmente, dos objetivos: por un lado, ofrecer experiencias pedagógicas positivas a lo largo de la vida en un contexto en el que - tal y como dice Judy Wajcman (2016) — las nuevas tecnologías pueden mantenernos siempre ocupados y, por el otro, prometer la adquisición de aprendizajes medibles que avalarán la consecución de competencias profesionales acreditadas por múltiples servicios educativos. La propuesta desescolarizadora de Ivan Illich (2011) parece haber encontrado, así, una realidad virtual en la que desarrollarse, aunque las tramas de aprendizaje emancipadas de las aulas con las que había soñado el autor austríaco, bastante más rudimentarias que las que brinda hoy una simple conexión a internet como lugar de producción de los intercambios y los afectos, tienen poco que ver con la tecnoutopía imaginada desde Silicon Valey.

Puesto que la arquitectura del nuevo modelo pedagógico se anclará en la economía política del mundo digital, la fórmula es conocida. La hemos apuntado antes y ya forma parte de las ofertas de aprendizaje en los estudios superiores, que marcan 
la hoja de ruta. Su justificación didáctica, basada en el principio doctrinal de que el alumno es el agente activo y responsable de su propio proceso de aprendizaje, es ampliamente aceptada. En un contexto hiperconectado, pues, hay que prever que el alumno pase cada vez menos tiempo con sus profesores - abocados desde hace tiempo, tal y como denuncia Giroux (2019), a un proceso imparable de proletarización-, porque serán datos procesados por algoritmos los que crearán playlist educativas personalizadas, adaptadas a sus gustos y comportamiento virtual. Estas playlist tomarán el relevo a los ladrillos prefabricados con los que el encorsetamiento curricular de la instrucción escolar - por seguir con la apropiación tech-libertarian de Illich - había troceado las disciplinas y las materias, esa institucionalización del saber, piedra angular de todo un sistema que supone también la institucionalización de la enseñanza, de los niveles educativos y de la asistencia a clase. En esa nueva estructura del saber, más horizontal y democrática, los profesores serán concebidos como organizadores y mediadores del encuentro de aprendizaje y gestores de los conocimientos a transmitir, que podrán ser de producción propia o ajena e incorporarán todo tipo de productos sofisticados, lenguajes y tecnologías — donde no se incluye el libro de texto-, gracias a los avances de la inteligencia artificial (IA). Desacreditado su oficio en tanto que deben convertirse en una especie de mediadores, coaches, animadores de aula o gestores de aprendizajes (Larrosa y Rechia, 2018, p. 451), ni tan solo serán necesarios para llevar a cabo el acompañamiento en el estudio puesto que podrán ser substituidos por robots. No es ciencia ficción. En estos momentos, se está invirtiendo mucho dinero en el diseño de software y todo tipo de prototipos tecnológicos basados en el internet de las cosas a fin de asistir los procesos de aprendizaje de los usuarios que ofrecerán las diferentes plataformas educativas. Pequeños robots virtuales, aplicaciones de autoevaluación, chatbots, etc., interactuarán con los estudiantes para alentar sus avances en un entorno de gamificación generalizada del aprendizaje controlado mediante algoritmos dispuestos a satisfacer su curiosidad en función de sus acciones e interacciones. ${ }^{2}$ Finalmente, la conectividad entre diferentes nodos de conocimiento, tal y como sucede con los populares MOOCs (Massive Online Open Courses, o cursos online masivos y abiertos) (Kiers, 2016), no hará más que multiplicar la oferta formativa y las posibilidades de expansión de un mercado que augura grandes beneficios.

Así las cosas, el desarrollo de tecnologías educativas e infraestructuras digitales no solo está renovando las estrategias formativas de la escuela con todo tipo de iniciativas (educación disruptiva, aula invertida, hiperaula, conectivismo, etc.), sino

2. En una entrada del blog de El Diari de l'educació, el profesor Francesc Imbermón apuntaba que en Cataluña existen «610 empresas dedicadas a las tecnologías educativas (infraestructuras, equipamiento, servicios de consultoría, relativas a los contenidos y recursos de aprendizaje, software, plataformas, etc.) y que, actualmente, representan el 1'03\% del PIB de Cataluña y va en aumento con el tratamiento de datos, aprendizaje a través de videojuegos, material, etc.» (Imbermón, 2019). 
que está logrando configurar una industria de servicios educativos personalizados que amenaza claramente la estructura actual del sistema educativo, considerado desde hace mucho tiempo como un sistema burocrático, costoso e ineficaz. ¿Para qué se va a financiar un sistema público de educación si lo pueden proveer las multinacionales tecnológicas y las empresas de comunicación?, se preguntan los más confiados. ${ }^{3}$ En la medida que "la educación — tal y como sostienen Fernández Liria, García Fernández y Galindo (2017, p. 37) — está dejando de concebirse como un derecho de ciudadanía y está empezando a transformarse en un servicio y en una inversión», esa lógica no solo rige el interés de las empresas y un sinfín de instituciones dispuestas a ofrecer todo tipo de servicios formativos avalados por el presunto prestigio de sus marcas, sino el de los propios usuarios, quienes se ven obligados a consumir esos servicios a fin de obtener aquellas competencias que favorezcan su empleabilidad o les permitan mantenerse actualizados para el desempeño de su trabajo.

\section{LA PEDAgOgía de LAS COMPETENCIAS}

Entramos así en el otro pilar de la transformación educativa, y es que nada de esto es ajeno a la promoción de un nuevo tipo de subjetividad, materializada en la figura del emprendedor (Barroso, 2015), que requiere el desarrollo de procesos formativos capaces de operar en un plano psicológico e instrumental. Sin duda, el discurso pedagógico en torno a las competencias cabe situarlo, sobre todo, a este nivel. Aunque incluye muchas otras cosas, la apuesta por un currículum competencial se considera un medio para favorecer el cambio de mentalidad que necesita la sociedad europea para adaptarse a los requerimientos del siglo XXI, donde prevalece un enfoque socioeconómico que se identifica con la mejora de las capacidades productivas y el desarrollo de las habilidades que permitirán generar un entorno competitivo. Así pues, la pedagogía de las competencias se presenta hoy como una innovación que debe potenciar unos aprendizajes más integrados, más prácticos y más transferibles, encontrando su razón de ser en la finalidad económica que se atribuye hoy en día a la educación.

3. Mientras escribo este artículo, la Fundación Telefónica acaba de anunciar la apertura del "plazo de inscripción de la denominada 'Escuela 42', una iniciativa que pretende "reinventar la educación” y aportar una alternativa «a un sistema educativo reglado incapaz de adaptarse a las demandas del mercado digital». Si bien no inventan nada que no ofrezcan ya los títulos oficiales de muchas universidades virtuales y otras plataformas educativas, sus promotores se apresuran en destacar que «la propuesta educativa carece de certificación y no requiere la presencia del profesor tradicional porque los alumnos aprenden a través de proyectos, que corrigen sus propios compañeros con el apoyo de un equipo pedagógico». Se puede acabar de leer la noticia en el periódico digital elEconomista.es. Recuperado de: https://www. msn.com/es-es/dinero/formacion-empleo/telef\%C3\%B3nica-propone-reinventar-la-educaci\%C3\%B3ndigital-colaborativa-gratuita-y-sin-profesores/ar-AACjUXo (Consultado el 18/06/2019). 
El discurso de la competencia ha recibido un fuerte apoyo entre el profesorado. Si lo defienden, incluso, los movimientos de renovación pedagógica es porque, de entrada, supone un cambio en la cultura escolar y universitaria al pasar de la cultura de la enseñanza y la instrucción directa a la cultura del aprendizaje, que tiene como protagonista al alumno y el desarrollo de sus capacidades, permitiendo la puesta en marcha de múltiples estrategias educativas basadas en las metodologías activas, colaborativas y experienciales que conectan muy bien con la tradición innovadora en el campo de la educación. Asimismo, hay quien ve en el enfoque educativo por competencias — quizás haciendo una lectura demasiado benevolente de la agenda política de la OCDE en materia de educación, inspiradora del modelo- una oportunidad para incorporar la educación en el desarrollo crítico y transformador (Egea, Massip, Flores y Barbeito, 2018).

Según la teoría de las competencias, el énfasis de la educación y la evaluación debe pasar del conocimiento a las actitudes. ¿Qué es lo que se considera pertinente aprender, entonces, en las escuelas? El discurso dominante insiste, tal y como hemos dicho, en la necesidad de adquirir habilidades transferibles al mercado de trabajo. Puesto que no hay una relación directa entre las competencias académicas y las competencias profesionales, habrá que centrar la atención en un saber hacer operativo y, sobre todo, actitudinal, de acuerdo con las necesidades expresadas desde las empresas o los mercados laborales y profesionales. Así, los títulos académicos deben acreditar un carné de competencias que servirá para que cada cual se lance a un mercado competitivo y global en el que poder demostrar aquellas capacidades y «conductas observables que se presuponen necesarias para el desempeño de un puesto de trabajo» (Fernández Liria, García Fernández y Galindo (2017, p. 97). Entre las más valoradas, destacan capacidades como la creatividad y la innovación, la adaptabilidad a las necesidades de las empresas, la flexibilidad, la iniciativa emprendedora, señalando las formas de actuar de las personas para activar nuevos mecanismos empresariales en detrimento de aquellos aprendizajes que podrían nutrir una verdadera formación ciudadana.

El discurso en torno a las competencias cuenta, como es obvio, con su aval psicopedagógico. Para ello, toma como referencia la teoría de las inteligencias múltiples, formulada por Howard Gardner (1994), cuyo principio fundamental es la atención a la diversidad a fin de personalizar la enseñanza en función de las fortalezas y debilidades del alumnado de acuerdo con sus múltiples capacidades, no solo la verbal y lógico-matemática. En un contexto de escuela inclusiva, se espera que esta sea capaz de respetar los ritmos singulares de desarrollo individual y de aprendizaje, ofreciendo las mismas oportunidades para promover el talento de todos (Muntaner, Rosselló y De la Iglesia, 2016). Esto supone, entre otras cosas, desplazar un currículum demasiado centrado en la inteligencia académica e intelectual hacia otro tipo de capacidades. Se trata, siguiendo a Gardner, de promover una escuela centrada en el individuo, cuyo objetivo «debería ser el de desarrollar las inteligencias 
y ayudar a la gente a alcanzar los fines vocacionales y aficiones que se adecuen a su particular espectro de inteligencias» (Gardner, 2015, p. 30).

De acuerdo con esta visión de las inteligencias múltiples, que han sido clasificadas de diversas maneras, diferentes autores defienden la necesidad de que la escuela se preocupe por el desarrollo de la inteligencia emocional (FernándezBerrocal y Ruiz, 2008; Bisquerra, Pérez-González y García Navarro, 2015). Según Daniel Goleman (1996), padre de esta teoría, la inteligencia emocional se presenta como la competencia para gestionar las emociones, orientar y desarrollar la propia vida y establecer relaciones constructivas con los demás. Estamos hablando, por lo tanto, de competencias que hacen referencia a capacidades relacionadas con la mejora personal y el establecimiento de relaciones positivas. En este sentido, tomar conciencia de la propia realidad emocional permite aplicar las estrategias más adecuadas para el desarrollo personal y profesional. Así pues, y tal y como sostienen Fernández Liria, García Fernández, y Galindo (2017, pp. 100-101, cursivas en el original),

mientras la inteligencia emocional determina nuestra capacidad para aprender los rudimentos del autocontrol y similares, la competencia emocional se refiere a nuestro grado de dominio de esas habilidades» de un modo que acabará reflejándose en el ámbito social y laboral. En este sentido, «el dominio de una determinada competencia emocional [...] requiere el desarrollo de algunas de las habilidades subyacentes a los principios fundamentales de la IE Emocional.

e ahí que hoy en día se hable tanto de la necesidad de sensibilizar al profesorado, a la Administración pública y a la sociedad en general en torno a la educación emocional del alumnado.

Quienes defienden la educación emocional, sostienen que «es una respuesta a las necesidades sociales que no están suficientemente atendidas en las áreas académicas ordinarias» (Bisquerra, 2018, p. 156). Asimismo, se afirma que existen evidencias que demuestran que la formación en competencias emocionales contribuye a mejorar la convivencia, el rendimiento y el bienestar. Prevenir el analfabetismo emocional que nos rodea sería uno de los objetivos de la educación emocional, que hay que entender como «un proceso educativo, continuo y permanente [...] a fin de capacitar para la vida y aumentar el bienestar personal y social» (2018, p. 157). Ahora bien, ¿se trata solo de eso? ¿Qué efectos subjetivos tiene la educación emocional? Abrir este interrogante nos permite hacer otras lecturas, poner en cuestión las bases desde las que esta se despliega cuando descubrimos que lo fundamental no se juega en el terreno de los aprendizajes. En este sentido, a nosotros nos interesa explorar qué hace la educación emocional a la hora de producir ciertas subjetividades acordes con lo que Laval y Dardot (2013) han venido a denominar como la nueva razón del mundo. Lo desarrollaremos en los siguientes apartados. 


\section{UN NUEVO PARADIGMA: LA NEUROEDUCACIÓN}

Realizar hoy en día una crítica de la educación emocional cuando cuenta con el consenso de tantos educadores y educadoras, parece un poco arriesgado. No faltan las voces que defienden, incluso, que se incluya en su propia formación profesional (Palomera, Fernández-Berrocal y Brackett, 2008; López-Goñi y Goñi, 2012; Body, Ramos, Redondo y Pelegrina, 2016; Darder, 2017). De hecho, «una de las corrientes pedagógicas que mayor presencia está teniendo en los nuevos discursos y prácticas educativas y que, por ello, es susceptible de fomentar nuevas identidades docentes, es el coaching educativon (Valero, 2019, p. 272; cursiva en el original). Sin embargo, nosotros quisiéramos llamar la atención — tal y como hemos apuntado- sobre los efectos de esta emopedagogía en el discurso educativo actual. En un trabajo anterior ya hablamos de la colonización Psi del sistema educativo (Solé y Moyano, 2017). Nos parece importante retomar aquella reflexión para analizar cómo va penetrando esta nueva ideología en el conjunto del sistema.

Desde que Édouard Claparède inaugurara el primer instituto de ciencias de la educación, dando nacimiento a la psicopedagogía a fin de prescribir los comportamientos de los educadores a partir del conocimiento científico de los niños, el discurso pedagógico está impregnado de lenguaje psicológico. Desde hace algunos años, además, conceptos como felicidad, necesidades emocionales, competencias sociales, motivación, autoestima forman parte de la vulgata pedagógica más extendida. Junto a ello, se han instaurado multitud de herramientas de evaluación, tablas de observación, protocolos, etc., con los que se diagnostican todo tipo de trastornos del aprendizaje, que cuentan ya con un amplio mercado de etiquetas y clasificaciones nosográficas (Morel, 2015), con sus tratamientos estandarizados, su derivación a dispositivos de externalización especializados y su batallón de expertos. La muestra más evidente en esa gestión tecnocrática de las diferencias sería, por ejemplo, la sobrediagnosticación del TDAH —en España ya se habla de la existencia de 400.000 niños diagnosticados con el TDAH—, ese cajón de sastre, tan rentable para una psicoindustria plenamente integrada en el Estado del bienestar, con el que se clasifica el malestar de la infancia en torno a la educación (Ubieto, 2014; Ubieto y Pérez Álvarez, 2018). Al respecto, Meirieu apunta que vivimos una época marcada por la hegemonía del psicologismo. Así, «ante la menor situación crítica se moviliza a los psicólogos y cuando, en una institución, aparece un conflicto, desde el primer momento se busca la fuente en los problemas de autoestima antes que... ¡en la lucha de clases! (Meirieu, 2016, p. 87).

Poco a poco, asistimos a un proceso de conversión de las cuestiones educativas - tal y como sucede en un orden social, político y cultural mucho más amplio- a cuestiones médicas e individuales cuando aparecen problemas disciplinarios, tanto en la adquisición de los aprendizajes como en el comportamiento de los alumnos en las aulas. Así, las diferencias acaban derivando en una pedagogía de las identidades patológicas, anulando la subjetividad (Leite y Christofoletti, 2018). Sin duda, 
en esa conversión intervienen diversos factores. Entre ellos, hay que destacar las transformaciones de los campos científico y médico y su desplazamiento hacia el paradigma positivista y empirista (tecnologías de cosificación, clasificación, medición, observación empírica, evaluación, etc.), reforzado por el impulso de las neurociencias cognitivas, que han penetrado con fuerza en el mundo de la educación (Ocampo, 2019). En poco tiempo, la neuroeducación, impulsada por la misma OCDE (2007), se ha convertido en un paradigma psicopedagógico dominante, hasta el punto que hay quien llega a afirmar la necesidad de rechazar cualquier propuesta educativa que no tenga «una base empírica verificada por la neurociencia» (Guillén, Pardo, Forés, Hernández, y Trinidad, 2015, p. 51), aunque sus aplicaciones prácticas sean más bien limitadas o no hayan hecho más que corroborar lugares comunes pedagógicos a la hora de apoyar, mediante evidencias empíricas, una escuela con cerebro.

Como es sabido, el ser humano no vive solo de ciencia, necesita también hogares mitológicos. Tal vez por eso la ciencia crea sus propios mitos; en este caso, los neuromitos (Ferrero, Garaizar y Vadillo, 2016). Si observamos de cerca algunos de los descubrimientos de la neurociencia, hallaremos que no hacen más que presentar con gran solemnidad científica ideas populares enraizadas en nuestra cultura sobre la psique, el bienestar o la salud humanas a partir de la objetividad que es capaz de proporcionar una simple radiografía. Sabemos muy bien la fascinación que despiertan las imágenes tomográficas del cerebro, las gráficas y cálculos matemáticos y los marcadores de actividad cerebral vistos desde la pantalla de un ordenador. En esas supuestas evidencias, donde una resonancia magnética funcional (fMRI) y un software informático se convierten en metáforas de la mente, confundiendo la actividad mental con las reacciones químicas y eléctricas del cerebro (Castoriana, 2016), reside todo su misterio, generando así una nueva construcción científica y, por tanto, un nuevo régimen de verdad que se acabará integrando exitosamente en el tejido social. El uso de técnicas estadísticas se encargará de ofrecer muestras empíricas definitivas, y es así como la traslación de esa investigación neurológica al sistema de diagnóstico de trastornos del aprendizaje y al diseño de programas cognitivo-conductuales para fomentar el bienestar requerirá de un simple giro psicopedagógico. Teniendo en cuenta que «la corteza cerebral tiene un potencial ilimitado de cambio, [...] cada persona es la arquitecta de su cerebro» (Bisquerra, 2018, p. 154). De esta forma, reformas curriculares y un sinfín de pautas, consejos y técnicas psi basadas o directamente extraídas de la literatura científica para ejercitar el cerebro en la autogestión emocional, incluyendo los tratamientos farmacológicos, empiezan a formar parte de las prácticas y rituales de muchas aulas (De Vos, 2015), a menudo justificadas por el noble objetivo de estimular y potenciar aquellos estilos y competencias educativas que mejor sintonizan con la manera de trabajar del cerebro a la hora de fomentar la motivación, controlar el comportamiento y mejorar los aprendizajes (Mora, 2013; Bueno, 2017). 


\section{El DESARROLlO de LA EDUCACIÓN EMOCIONAL}

Junto a las aportaciones de la neuroeducación, el desarrollo de programas de educación emocional se ha visto también amparado por el avance - en términos culturales y comerciales - de la psicología positiva o ciencia de la felicidad, un movimiento académico nacido a principios de este siglo dispuesto a defender la existencia de unos factores psicológicos clave para ayudar a la gente a llevar una vida más feliz (Csikszentmihalyi, 1997).

No disponemos de suficiente espacio para trazar una geneología de la psicología positiva, promovida desde la poderosa Asociación Estadounidense de Psicología (APA, en sus siglas en inglés) con el objetivo de fundar un campo de estudio nuevo y alternativo respecto de la psicología tradicional, basada en un modelo patológico del comportamiento humano (Seligman y Csikszentmihalyi, 2000). Solo hay que retener el hecho de que rasgos psicológicos como la autoestima, la inteligencia emocional, la autonomía, el optimismo, la resiliencia, la proactividad, el autocontrol, etc., han pasado a convertirse en contenidos pedagógicos esenciales de estos programas, cuyo objetivo es la autorrealización, el crecimiento personal y la felicidad. Tal y como afirma Cabanas (2019, p. 243),

Las escuelas y las instituciones de educación superior son ámbitos para la aplicación de intervenciones psicológicas positivas sobre inteligencia emocional, emociones positivas, pensamiento positivo, resiliencia, optimismo y automotivación; también son campos donde el sesgo positivista e individualista de las intervenciones basadas en la felicidad es particularmente manifiesto.

Una amplia oferta de intervenciones y programas de educación positiva, entrenamiento autoinstruccional y autogestión emocional se están instaurando en muchos centros educativos, donde han hallado una "cultura educativa cada vez más interesada en las competencias emocionales, en las habilidades de gestión personal y en la promoción del espíritu emprendedor» (Cabanas e Illouz, 2019, p. 84), conscientes de ser el primer estadio de una inversión en capital psicológico $y$ emocional que no solo contribuye a aumentar el bienestar y la felicidad de los alumnos, sino que los ayuda a expresar su talento para alcanzar el potencial que les permitirá aumentar sus posibilidades de éxito en todos los ámbitos de la vida. Tal y como sostienen los más entusiastas,

garantizar la presencia de la dimensión afectiva dentro de los cambios que se están realizando en los sistemas educativos significa poner el énfasis en el pleno desarrollo de las personas y en la construcción de una ciudadanía activa y emprendedora que persigue la mejora de todo el colectivo (Darder, 2017, p. 16).

Desde la escuela hasta la universidad asistimos, pues, a una priorización de las emociones en el aprendizaje en todo tipo de propuestas de formación, tutoría, programas para los diferentes niveles educativos, etc. (Bisquerra, 2010, 2011; 
Carpena, 2017; GROP, 2009; Güell y Muñoz, 2003; Sánchez, Rodríguez y García, 2018). A menudo se justifica por el hecho de que vivimos un momento de cambios muy profundos y acelerados que presionan sobre el equilibrio personal y la vida social y que, por ese motivo, hay que fortalecer la vida afectiva de los individuos. Desde un punto de vista pedagógico, existe también un acuerdo generalizado en considerar que muchos problemas de aprendizaje tienen su origen en disfunciones emocionales y afectivas (Darder, 2017, p. 16). Sea como fuere, todo ello se desarrolla en un contexto vinculado con el creciente valor cultural de las emociones en la constitución de la propia identidad, las relaciones sociales y el propio bienestar (Prieto, 2018).

Un ethos terapéutico que encaja muy bien con los valores promovidos por la cultura neoliberal se está imponiendo, pues, en todos los niveles del sistema educativo. Solo ha hecho falta asumir que la plena funcionalidad y valor de cada uno como individuo depende de su constante optimización personal mediante la adquisición de habilidades de gestión emocional y cognitiva y el desarrollo pleno de sus capacidades. Aprender a manejar, contener y canalizar las emociones forma parte, entonces, de un trabajo psicopedagógico necesario al servicio de la maximización del interés personal.

En este contexto de utilitarismo emocional, la psicología positiva, el coaching y la literatura de autoayuda, que se encuentran en la base de una educación emocional new age cada vez más extendida (Malagón, 2011; López y Valls, 2013; Sánchez y Boronat, 2014; Sandoval y López, 2017), no hacen más que promover - a nuestro entender - un tipo de educación integral abocada al culto narcisista. "En la educación —afirma Darder (2017, p. 17) — es indispensable favorecer el nivel de conciencia (de nosotros mismos, del entorno, de los otros y del contexto y los vínculos establecidos), para que cada uno asuma el gobierno de sí mismo». En este sentido, los programas de educación emocional se encargan de ofrecer todo tipo de estrategias - una suerte de tecnologías del yo, por usar el término de Foucault (2008)_, así como un modo emocional de reflexividad para que cada cual aprenda a gestionar su mundo interior, refuerce su autoestima y capacidad de resiliencia, confronte sus emociones negativas y explore el fantasma de su libertad; un fantasma que se apoya en el mito de la expansión de uno mismo, el crecimiento personal y la potenciación de la propia individualidad como el camino más seguro para alcanzar la felicidad. Y es que la felicidad — tal y como sostienen Cabanas e Illouz (2019, p. 123) — se ha convertido «en un estilo de vida, en una mentalidad y, en último término, en un tipo de personalidad para definir en términos psicológicos el ideal neoliberal del ciudadano contemporáneo».

\section{EFECTOS SUBJETIVOS DE LA EDUCACIÓN EMOCIONAL}

En efecto, la felicidad juega hoy un papel crucial para obtener el control de nuestras subjetividades y moldear el arquetipo ideal del ciudadano neoliberal. La 
felicidad — tal y como sostiene Ehrenreich (2009) — marca la norma de lo que es bueno, sano y deseable, tanto para el individuo como para las sociedades. Por este motivo, la educación emocional se ha convertido en una pieza angular de las nuevas formas de individualismo que promueve el neoliberalismo. Imbuidas de un discurso altamente empresarial, las técnicas, métodos y actividades que desarrollan los programas de psicología positiva en escuelas, institutos y universidades ofrecen así una caja de herramientas para llevar a cabo un trabajo afectivo muy estandarizado a fin de interiorizar la persistente invocación neoliberal a una vida regida por la satisfacción de los propios intereses, el crecimiento personal, la gratificación emocional y la competitividad, objetivos prioritarios de una formación que - tal y como sostiene Recalcati (2016, p. 35) — acaba siendo «reducida a la mera potenciación del principio del rendimiento» con el objetivo de preparar a las nuevas generaciones "para la implacable competición de la vida".

Desde hace unos años, se ha instalado una corriente de opinión en el mundo educativo que insiste en el desarrollo de una pedagogía de las emociones. No es una pedagogía que se preocupe tan solo por el desarrollo emocional de los niños, asumido desde todas las corrientes educativas que han aceptado las tesis constructivistas, mayoritarias en el sistema educativo, sino, sobre todo, por su felicidad y, por tanto, por los caminos que hay que seguir para conseguirla.

En un momento en el que la promesa de la educación ha caído, esta ha sido substituida por la educación que promete la felicidad a través de la educación emocional, dispuesta a proyectar los valores morales y los factores psicológicos clave que subyacen a la búsqueda de la felicidad. Una felicidad que - tal y como afirma Cabanas (2019, p. 237) — no hay que entender tan solo como una emoción o un estado psicológico, sino como «un tipo de subjetividad específico y normativo que se define intensa y predominantemente en términos y prácticas emocionales y psicológicos".

La subordinación de la tarea educativa al desarrollo y florecimiento de las emociones, que se han convertido en objetos naturalizados que pueden ser transformados y manejados mediante acciones estratégicas al margen de cualquier contenido cultural, que es lo que cabe esperar que transmita la escuela, no puede esconder el vacío que se esconde tras la promesa individualista de la felicidad. Siendo así, la propuesta cultural de la educación queda abocada a la transmisión de ese vacío.

Desplazados los contenidos culturales, así como las actividades pedagógicas que requieren repetición, tiempo y esfuerzo, asistimos a una intensificación de la vida emocional en la escuela que se manifiesta de muchas maneras. Las acciones educativas legítimas son aquellas que tienen en cuenta las emociones de los niños y las niñas. La orientación de los alumnos se encamina en función de proyectos emocionales que refuerzan su identidad. Mientras tanto, el respeto a los intereses de los estudiantes se lleva a cabo sin cuestionar las motivaciones e intenciones emocionales determinadas por la cultura del consumo que, como es sabido, trabaja 
desde el núcleo mismo de los guiones culturales de la individualidad, estimulando reclamos morales de vida personal, autenticidad, autonomía y realización personal.

Sin duda, todo ello conecta muy bien con los procesos de enculturación de las jóvenes generaciones, habituadas a seguir a todo tipo de influencers, youtubers e instagramers dispuestos a transferir experiencias, emociones y estilos de vida a través de las redes sociales (Guardiola, 2018, p. 54). El marketing emocional forma parte hoy de un gran simulacro global transmitido a través de la superficie ilusionante de las múltiples pantallas a las que estamos enganchados.

En este contexto, el mundo educativo asume acríticamente un discurso favorable a las competencias emocionales porque las sociedades occidentales somos, sobre todo, sociedades psicológicas. El discurso en torno a las emociones y la felicidad forma parte de nuestro repertorio cultural, basado en un «modelo de ciudadanía altamente individualista y psicológico» (Cabanas, 2019, p. 236). Y es así como nos concebimos a nosotros mismos siguiendo marcos simbólicos que se nutren de la psicología. No nos reconocemos, como individuos, en la visión que puedan ofrecernos las categorías morales o religiosas, ni tan solo en elementos culturales tradicionales con los que a menudo se explican las sociedades de pertenencia, sino a través de nuestras emociones, que la psicología ha ayudado a identificar para entender el valor y el significado de nuestras conductas, nuestras relaciones, nuestros deseos y sentimientos sociales, así como nuestro inconsciente, nuestros síntomas, nuestros estados de ánimo, etc. Es a través de todo ello que logramos construir una narrativa en torno a nuestra propia identidad.

Todos consumimos, practicamos y reproducimos modelos psicológicos de emociones. Las hemos integrado en nuestro léxico verbal, apropiándonos, incluso, de conocimiento experto, a un clic de distancia de nuestra ignorancia, para exponer nuestra identidad social. Asimismo, nos prestamos a identificar nuestra vida emocional y a remodelar nuestra composición psicológica a través de proyectos emocionales: reducir la depresión, aliviar el estrés, la ira o la ansiedad, hallar paz interior, decidir el futuro de una relación de pareja o una carrera profesional, promover la autoestima, la satisfacción, el bienestar, construir una personalidad auténtica, etc.

El discurso terapéutico lo hallamos, además, en el mundo del trabajo. Eva Illouz (2007, p. 19) ha demostrado hasta qué punto ese discurso ha sido necesario para la reorganización de la imaginación empresaria. En efecto, desde hace mucho tiempo, el management empresarial utiliza el trabajo emocional de los trabajadores como un elemento cultural de producción de valor de las empresas (Boltanski y Chiapello, 2002); de hecho, tal y como sostiene Biung-Chul Han (2014) en Psicopolítica, las emociones se han convertido en el recurso necesario para incrementar la productividad y el rendimiento. En un mundo económico y profesional altamente individualizado en el que el capitalismo se efectúa "mediante la movilización de nuestras vidas» (López Petit, 2009, p. 63), las emociones contribuyen a mejorar, en definitiva, el branding personal, la marca de ese empresario de sí -al decir de 
Foucault (2007)—, que no puede dejar de invertir en las propias posibilidades de éxito, satisfacción y empleabilidad.

El mismo proceso lo encontramos también en el reino del consumo, otro elemento clave en la construcción de la subjetividad y las relaciones sociales, donde se busca con avidez experiencias emocionales gratificantes mediante la adquisición de objetos y rituales. Finalmente, el individuo y sus emociones se han convertido en los objetivos más preciados de una industria que vende salud mental, crecimiento personal y bienestar emocional en la que podemos encontrar todo tipo de investigaciones científicas, teorías académicas, productos farmacéuticos, aplicaciones tecnológicas de felicidad y prácticas psicológicas. Desde el psicoanálisis a las terapias breves y humanistas, pasando por el Neurofeedback, los tratamientos cognitivoconductuales, las técnicas de psicología positiva más elementales, el coaching, la literatura de autoayuda, así como la gran diversidad de prácticas imaginarias y corporales dirigidas a públicos muy variados, como el yoga y el mindfulness, pero también la psicomagia y formas populares de misticismo oriental, chamanismo opiáceo y espiritualidad, estamos inmersos en un amplio mercado de automodelado emocional y cognitivo como objetos de preferencia para el consumo individual y de estilos de vida (Illouz, 2019).

\section{RESTAURAR EL COMPROMISO PEDAGógico}

No descubriremos ahora hasta qué punto el capitalismo ha integrado las experiencias emocionales para consolidar una subjetividad conformada a sus exigencias. De hecho, también las produce a fin de crear un yo y una identidad psicológicos privados, alentando el individualismo y el repliegue del sujeto en sí mismo. La psicología ha reforzado la idea de que las emociones son entidades que se pueden gestionar a fin de alcanzar una autenticidad emocional con la que definir la propia identidad. La industria psicomédica, por su parte, ha intensificado la cosificación de las emociones, estableciendo categorías objetivas que se pueden llegar a conocer mediante la investigación científica del cerebro y, en su caso, medicalizar. Para ello, el DSM y las versiones más actualizadas de los manuales de psiquiatría ya se encargan de patologizar todo tipo de emociones y conductas. El mercado de los trastornos y enfermedades nuevas es infinito. En este contexto, la educación emocional, orientada a aumentar la experiencia de estados de ánimo y emociones positivas, deviene, junto al sector psi, un actor clave en la estructuración del orden social y moral capitalista.

Nos hallamos, pues, ante un proyecto de modelización subjetiva, y es que la educación emocional parece haber capturado el sentido último de la política cultural del neoliberalismo. En su empeño por estimular un yo positivo y exitoso, la forma más acabada del individualismo radical y emprendedor se aparta del verdadero compromiso pedagógico, institucionalizando el lenguaje y la práctica de la psicoterapia en nombre del crecimiento personal y la felicidad de los alumnos, obviando el 
hecho de que estos valores o metas sociales han servido para neutralizar y legitimar la ideología neoliberal del individualismo (Cabanas e Illouz, 2019).

¿Cuál es, pues, el compromiso pedagógico de la educación emocional? Sin duda, y aquí coincidimos con Darder (2017, p. 21), "prescindir de las emociones es una simplificación que mutila la complejidad de la realidad y de la educación. Las vivencias emocionales y afectivas del sujeto intervienen y condicionan su desarrollo personal y social y, por tanto, el aprendizaje», pero estamos hablando de otra cosa. Desde nuestra perspectiva, la función educativa capaz de sostener el compromiso pedagógico es aquella que — al decir de Hannah Arendt (1996) — se hace cargo de abrir el mundo a las nuevas generaciones; hacer que el mundo (lo que hay más allá de uno mismo y de la potenciación productiva de su talento), les hable y les interpele. $\mathrm{Y}$ aquello que les debe interpelar - tal y como defienden Masschelein y Simons (2014, p. 27)—, tiene que ver con el aprendizaje, con la iniciación al conocimiento y a las destrezas, con la socialización en la cultura de una sociedad entendida en un sentido amplio, y no con la exaltación emotiva del yo y su autonomía, objetivo prioritario de las agendas narcisistas de la cultura terapéutica del neoliberalismo.

Si nos apartamos del enfoque psicologizante de la educación y nos ponemos a trabajar a partir de la cultura, "la única materia de la enseñanza escolar», tal y como dice Meirieu (2016, p. 72), dotamos a las jóvenes generaciones de «los lenguajes que les permitirán comunicarse, los instrumentos gracias a los cuales podrán hacer frente a los acontecimientos cotidianos, los conceptos y los modelos que les darán los medios de comprender lo que les rodea, lo que les pasa» (2016, p. 72). Defender el compromiso pedagógico supone comprender que la educación es el esfuerzo permanente por poner en contacto al sujeto con la cultura, partiendo de su particularidad, pero no encerrándose en ella.

Hay que cuestionar, pues, todo este discurso de la emopedagogía, así como el trabajo científico y académico que lo avala. La educación emocional es una falsa recompensa ante la falta de promesa en educación. Cuando la formación ya no garantiza nada, cuando los alumnos son muy conscientes del destino social que les espera (los méritos personales no se traducen en resultados), la educación emocional supone una falsa salida. Alejados de la cultura, la educación emocional, autocentrada en un yo vacío de saberes, les impide vivir el placer de los aprendizajes y las «satisfacciones del pensamiento» (2016, p. 83). Para que esto sea posible, es decir, para rehabilitar la promesa de la educación mediante el goce de aprender, «hace falta que el adulto [...] — tal y como sostiene de nuevo Meirieu (2016, p. 83)—, tome la posta de la promesa institucional: a él le corresponde encarnar, en su comportamiento de educador, el placer de investigar y la alegría de conocer». Ahí reside — siguiendo a Bárcena (2018, p. 79; cursivas en el original) — su función como «mediador del deseo de saber del otro". Subordinar los aprendizajes a las emociones desarma a los alumnos y refuerza las desigualdades, impidiendo el acceso a la única promesa 
educativa que hoy es posible sostener: "la promesa de que el esfuerzo intelectual permite acceder a la alegría de pensar» (Meirieu, 2016, p. 85).

No podemos sustituir nuestra responsabilidad pedagógica, es decir, despertar el interés de los jóvenes por los conocimientos y destrezas socialmente importantes que se despliegan a través de las materias escolares, esas ventanas abiertas al mundo, por una serie de prácticas aplicadas a la gestión psicoemocional de los talentos individuales. Esas prácticas se ven reforzadas, además, por las estrategias mercantilizadoras de una educación digital preocupada, tal y como hemos visto en el primer apartado, por el desarrollo exclusivo de competencias y habilidades profesionales. La educación es algo más que simple formación para el trabajo. La educación es también una oportunidad para que los jóvenes dejen de ser seducidos por algo que vaya más allá de sí mismos y de sus identidades particulares, en el que antes que la exaltación del individuo, erigido en valor soberano, cabe hallar el encuentro con el otro encarnado por el maestro, cuya función como mediador de la cultura, gracias a una interacción de tutela — al decir de Jerome Bruner (2013)—, prepara la apertura a nuevos mundos «a través de la materia colocada en medio de la relación» (Bárcena, 2018, p. 90), esos mundos que conforman nuestro mundo común y permiten instituir lo colectivo (Garcés, 2013). Para ello, sin duda, hace falta escuela (Larrosa, 2018), y no un simple menú de plataformas educativas a la carta.

Tal es, sin duda, el sentido de la enseñanza de Hannah Arendt cuando, en su conocido análisis sobre "la crisis de la educación", nos insta a "asumir la responsabilidad del mundo" hacia las nuevas generaciones: "Con la educación decidimos si amamos lo suficiente a nuestros niños para no apartarlos de nuestro mundo ni abandonarlos a sí mismos, [...], y, en cambio, prepararlos de antemano para la tarea de renovar un mundo común" (Arendt, 1996, p. 208). No abandonar, pues, a los alumnos a sí mismos en una pedagogía de las competencias y de las emociones, a menudo defendida por muchos educadores de una manera tan ingenua como peligrosa, si se la desconecta de un proyecto cultural fuerte traducido en actos de trabajo cotidiano que permitan acceder al pensamiento y, por lo tanto, a crecer y aprender.

\section{REFERENCIAS BIBLIOGRÁFICAS}

Arendt, H. (1996). Entre el pasado y el futuro. Barcelona: Península.

Bárcena, F. (2018). Maestros y discípulos. Anatomía de una relación. Teoría de la Educación. Revista Interuniversitaria, 30(2), 73-108. http://dx.doi.org/10.14201/teoredu30273108.

Barroso, C. (2015). Emprendedor: cuando el término es más que una palabra. En L. Núñez Cubero (Coord.), Cultura emprendedora y Educación (pp. 133-142). Sevilla: Universidad de Sevilla.

Bermejo, J. (2018). Plenitud personal e innovación educativa. Reflexiones en torno al deber educativo. Temps d'Educació, 55(1), 297-307. http://dx.doi.org/10.1344/TE2018.55.16.

Bisquerra, R. (Coord.) (2010). La educación emocional en la práctica. Barcelona: Horsori-ICE. 
JORDI SOLÉ BLANCH

EL CAMBIO EDUCATIVO ANTE LA INNOVACIÓN TECNOLÓGICA, LA PEDAGOGÍA DE LAS COMPETENCIAS Y EL DISCURSO DE LA EDUCACIÓN EMOCIONAL. UNA MIRADA CRÍTICA

Bisquerra, R. (2011). Educación emocional: Propuestas para educadores y familias. Bilbao: Desclée De Brouwer.

Bisquerra, R., Pérez-González, J. C. y García Navarro, E. (2015). Inteligencia emocional en la educación. Madrid: Síntesis.

Bisquerra, R. (2018). Educació emocional: de la recerca a la pràctica fonamentada. Revista Catalana de Pedagogia, 13, 145-171. Recuperado de: https://publicacions.iec.cat/repository/pdf/00000257/00000062.pdf (Consultado el 20/09/2019).

Body, L., Ramos, N., Recondo, O. y Pelegrina, M. (2016). Desarrollo de la inteligencia emocional a través del programa mindfulness para regular emociones (PINEP) en el profesorado. Revista Interuniversitaria de Formación del Profesorado, 87, 47-59. Recuperado de: http:// www.redalyc.org/jatsRepo/274/27449361006/html/index.html (Consultado el 20/09/2019).

Boltanski, L. y Chiapello, É. (2002). El nuevo espíritu del capitalismo. Madrid: Akal.

Bruner, J. (2013). La educación, puerta de la cultura. Madrid: Antonio Machado.

Bueno, D. (2017). Neurociència per educadors. Tot allò que els educadors sempre han volgut saber sobre el cervell dels seus alumnes i mai ningú s'ha atrevit a explicar-los de manera entenedora i útil. Barcelona: Associació de Mestres Rosa Sensat.

Cabanas, E. e Illouz, E. (2019). Happycracia. Cómo la ciencia y la industria de la felicidad controlan nuestras vidas. Barcelona: Paidós.

Cabanas, E. (2019). Psiudadanos, o la construcción de individuos felices en las sociedades neoliberales. En E. Illouz (comp.), Capitalismo, consumo y autenticidad. Las emociones como mercancía (pp. 233-263). Buenos Aires / Madrid: Katz Editores.

Carpena, A. (2017). Retalls de vida emocional: Educar la interioritat i la intel.ligència social. Vic: Eumo.

Castorina, J. (2016). La relación problemática entre Neurociencias y educación. Condiciones y análisis crítico. Propuesta Educativa, 46, 26-41. Recuperado de: http://www.redalyc. org/pdf/4030/403049783004.pdf (Consultado el 20/09/2019).

Csikszentmihalyi, M. (1997). Fluir (flow): Una psicología de la felicidad. Barcelona: Kairós.

Darder, P. (Coord.) (2017). La formació emocional del professorat. Barcelona: Octaedro.

De Vos, J. (2015). Deneurologizing Education? From Psychologisation to Neurologisation and Back. Studies in Philosophy and Education, 34(3), 279-295. Recuperado de: http://hdl. handle.net/1854/LU-5710070 (Consultado el 20/09/2019).

Egea, A., Massip, C., Flores, M. i Barbeito, C. (2018). Competències per transformar el món: cap a una educació crítica i per a la justícia global a l'escola. Barcelona: Graó.

Ehrenreich, B (2012). Sonríe o Muere. La Trampa del Pensamiento Positivo. Madrid: Turner.

Fernández-Berrocal, P. y Ruiz Aranda, D. (2008). La Inteligencia emocional en la educación. Revista Electrónica de Investigación Socioeducativa, 15(6), 421-436. Recuperado de: http://www.redalyc.org/pdf/2931/293121924009.pdf (Consultado el 20/09/2019).

Fernández Liria, C., García Fernández, O. y Galindo Fernandez, E. (2017). Escuela o barbarie. Entre el neoliberalismo salvaje y el delirio de la izquierda. Madrid: Akal.

Ferrero, M., Garaizar, P. y Vadillo, M. (2016). Neuromyths in education: Prevalence among Spanish Teachers and an Exploration of Cross-cultural variation. Frontiers in Human Neuroscience, 10(496), 1-11. http://doi.org/10.3389/fnhum.2016.00496.

Foucault, M. (2007). Nacimiento de la biopolítica. México: Fondo de Cultura Económica. 
Foucault, M. (2008). Tecnologías del yo y otros textos afines. Buenos Aires: Paidós.

Garcés, M. (2013). Un mundo común. Barcelona: Bellaterra, S.L.

Gardner, H. (1994). Estructuras de la mente: la teoría de las inteligencias múltiples. Madrid: Fondo de Cultura Económica.

Gardner, H. (2015). Inteligencias múltiples. La teoría en la práctica. Barcelona: Paidós.

Giroux, Henry A. (2019). La guerra del neoliberalismo contra la educación superior. Barcelona: Herder.

Goleman, D. (1996). Inteligencia emocional. Barcelona: Kairós.

Grup de Recerca en Orientació Psicopedagògica (GROP) (2009). Activitats per al desenvolupament de la intel.ligència emocional. Barcelona: Parramón.

Guardiola, I. (2018). L'ull i la navalla. Un assaig sobre el món com a interfície. Barcelona: Arcàdia.

Güell, M. y Muñoz, J. (2003). Educación emocional: Programa para la educación secundaria postobligatoria. Barcelona: Wolters Kluwer.

Guillén, J. C., Pardo, F., Forés, A., Hernández, T. y Trinidad, C. (2015). Principis neurodidàctics per a l'aprenentatge. Temps d'Educació, 49, 49-67. Recuperado de: https://www.raco. cat/index.php/TempsEducacio/article/view/303522/393207 (Consultado el 20/09/2019).

Han, Byung-Chul (2014). Psicopolitica. Barcelona: Herder.

Illich, I. (2011). La sociedad desescolarizada. Buenos Aires: Ediciones Godot.

Illouz, E. (2007). Intimidades congeladas. Las emociones en el capitalismo. Buenos Aires / Madrid: Katz Editores.

Illouz, E. (comp.) (2019). Capitalismo, consumo y autenticidad. Las emociones como mercancía. Buenos Aires / Madrid: Katz Editores.

Imbermón, F. (2019). Els apocalíptics de la tecnologia educativa; robots a les aules? El Diari de l'educació, 3 de junio. Recuperado de: http://diarieducacio.cat/els-apocaliptics-de-la-tecnologia-educativa-robots-a-les-aules/?utm_source=Subscriptors\&utm campaign=0fbc8b8a96-EMAIL_CAMPAIGN_2016_11_24_COPY_01\&utm_ medium=email\&utm_term=0_80c91da0fa-0fbc8b8a96-157775113 (Consultado el 20/09/2019).

Kiers, J. (2016). MOOCs and their Effect on the Institution: Experiences in Course Design, Delivery and Evaluation; Research; Faculty Development; Unbundling and Credits for MOOCs. Foro de Educación, 14(21), 133-149. http://dx.doi.org/10.14516/fde.2016.014.021.007.

Larrosa, J. (Ed.) (2018). Elogio de la escuela. Buenos Aires: Miño y Dávila.

Larrosa, J. y Rechia, K. (2018). P de profesor. Buenos Aires: Noveduc.

Laval, Ch. (2004). La escuela no es una empresa. El ataque neoliberal a la enseñanza pública. Barcelona: Paidós Ibérica.

Laval, Ch. y Dardot, P. (2013). La nueva razón del mundo. Ensayo sobre la sociedad neoliberal. Barcelona: Gedisa.

Leite, C. D. P. y Christofoletti, R. (2018). Childhood and education: between medicalization and subjectivation processes in school. Foro de Educación, 16(24), 113-123. http:// dx.doi.org/10.14516/fde. 583 . 
JORDI SOLÉ BLANCH

EL CAMBIO EDUCATIVO ANTE LA INNOVACIÓN TECNOLÓGICA, LA PEDAGOGÍA DE LAS COMPETENCIAS Y EL DISCURSO DE LA EDUCACIÓN EMOCIONAL. UNA MIRADA CRÍTICA

López-Goñi, I. y Goñi, J. M. (2012). La competencia emocional en los currículos de formación inicial de los docentes. Un estudio comparativo. Revista de Educación, 357, 205-206. https://doi.org/10-4438/1988-592X-RE-2010-357-069.

López Pérez, C. y Valls, C. (2013). Coaching educativo. Las emociones al servicio del aprendizaje. Barcelona: Ediciones S.M.

López Petit, S. (2009). La movilización global. Breve tratado para atacar la realidad. Madrid: Traficantes de Sueños.

Macluhan, M. (1986). El aula sin muros. Barcelona: Cultura Popular.

Malagón, F. J. (2011). Coaching educativo y académico: un nuevo modo de enseñar y aprender. Educación y futuro, 24, 49-66. Recuperado de: https://dialnet.unirioja.es/servlet/ articulo?codigo=3632848 (Consultado el 20/09/2019).

Masschelein, J. y Simons, M. (2014). Defensa de la escuela. Una cuestión pública. Buenos Aires: Miño y Dávila.

Meirieu, Ph. (2016). Recuperar la pedagogía. De lugares comunes a conceptos claves. Buenos Aires: Paidós Argentina.

Mora, F. (2013). Neuroeducación: sólo se puede aprender aquello que se ama. Madrid: Alianza.

Morel, S. (2015). La medicalización del fracaso escolar en Francia. Una forma contemporánea de etiquetaje de los alumnos con dificultades escolares. Revista de la Asociación de Sociología de la Educación, vol. 8, núm. 3, 321-334. Recuperado de: https://ojs.uv.es/ index.php/RASE/article/view/8387 (Consultado el 20/09/2019).

Morozov, E. (2015). La locura del solucionismo tecnológico. Madrid: Clave Intelectual.

Muntaner, Joan J., Rosselló, María R. y De la Iglesia, B. (2016). Buenas prácticas en educación inclusiva. Educatio Siglo XXI, 34(1), 31-50. https://doi.org/10.6018/j/252521.

Ocampo, J. Carlos (2019). Sobre lo «neuro» en la neuroeducación: de la psicologización a la neurologización de la escuela. Sophia, 26, 141-169. https://doi.org/10.17163/soph. n26.2019.04.

OECD (2007). Understanding the brain: the birth of a learning science. París: OECD.

Palomera, R., Fernández-Berrocal, P. y Brackett, M. A. (2008). La inteligencia emocional como una competencia básica en la formación inicial de los docentes: algunas evidencias. Revista Electrónica de Investigación Socioeducativa, 15(6), 436-454. Recuperado de: http://emotional.intelligence.uma.es/documentos/PDF67preservice_teacher_training. pdf (Consultado el 20/09/2019).

Prieto Egido, M. (2018). La psicologización de la educación: Implicaciones pedagógicas de la inteligencia emocional y la psicología positiva. Educación XX1, 21(1), 303-320, https:// doi.org/ 10.5944/educXX1.16058.

Recalcati, M. (2016). La hora de clase. Por una erótica de la enseñanza. Barcelona: Editorial Anagrama.

Sánchez, B. y Boronat, J. (2014). Coaching educativo: modelo para el desarrollo de competencias intra e interpersonales. Educación XXI, 17(1), 221-242. http://dx.doi.org/10.5944/ educxx1.17.1.10712.

Sánchez Calleja, L., Rodríguez Gómez, G. y García Jiménez, E. (2018). Desarrollo de competencias emocionales a través del Programa AEdEm para Educación Secundaria. Revista Complutense de Educación, 29(4), 975-994. https://doi.org/10.5209/RCED.54402. 
Sandoval, C. y López, O. (2017). Educación, psicología y coaching: un entramado positivo. Educatio Siglo XXI, 35(1), 145-164. http://dx.doi. org/10.6018/j/286261.

Seligman, M. E. P. y Csikszentmihalyi, M. (2000). Positive Psychology: An Introduction. American Psychologist, 55(1), 5-14. http://dx.doi.org/10.1037/0003-066X.55.1.5.

Solé Blanch, J. y Moyano Mangas, S. (2017). La colonización Psi del discurso educativo. Foro de Educación, 15(23), 101-120. http://dx.doi.org/10.14516/fde.551.

Ubieto, J. R. (2014). TDAH. Hablar con el cuerpo. Barcelona: Editorial UOC.

Ubieto, José R. y Pérez Álvarez, M. (2018). Niñ@s hiper. Infancias hiperactivas, hipersexualizadas, hiperconectadas. Barcelona: Ned Ediciones.

Valero Moya, A. (2019). Coaching educativo: ¿Qué identidad docente nos revela esta nueva corriente? Foro de Educación, 17(27), 271-287. http://dx.doi.org/10.14516/fde.657.

Wajcman, J. (2016). El temps a l'era digital. Barcelona: Centre de Cultura Contemporània de Barcelona. 
